\author{
Л.Н. Комарова*1,2, Р.Н. Ряхин², Ф.Ш. Алиев,2, С.А. Звезда \\ 1 - ФГБОУ ВО «Тюменский государственный медицинский университет» Минздрава РФ, Тюмень, Россия \\ 2 - НУЗ «Отделенческая больница на ст. Тюмень ОАО «РЖД», Тюмень, Россия
}

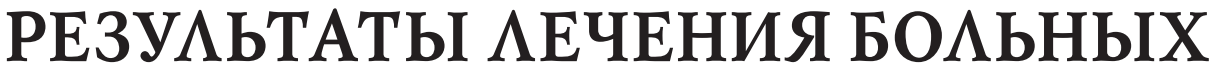 ВАРИКОЗНОЙ БОАЕЗНЬЮ НИЖНИХ КОНЕЧНОСТЕЙ ЗА 10 АЕТ
}

\author{
L.N. Komarova*1,2, R.N. Ryakhin'2, F.S. Aliev',2, S.A. Zvezda' \\ 1 - Federal State Budgetary Educational Institution of Higher Education «Tyumen State Medical University» \\ of the Ministry of Health of the Russian Federation, Tyumen, Russia \\ ${ }^{2}-\ll \mathrm{NGHCl}$ the branch hospital, Tyumen Station OJSC «Russian Railways», Tyumen, Russia

\section{TREATMENT RESULTS OF PATIENTS WITH VARICOSE VEINS DISEASE FOR TEN YEARS}

\begin{abstract}
Резюме
Цель: изучить качество жизни больных варикозной болезнью нижних конечностей, пролеченных за период с 2007 по 2016 годы оперативным путём, включая метод РЧА. Материалы и методы. В работе были использованы следующие материалы: истории болезни больного хирургического отделения, сводные данные из отдела статистики НУз «Отделенческая больница на ст. Тюмень ОАО «РЖД», данные ультразвукового ангиосканирования вен нижних конечностей. Использованные методы исследования: статистический метод, клинический, экспертный анализ и системный подход. Степень хронической венозной недостаточности нижних конечностей определяли с использованием клинического раздела международной классификации СЕАР (1995). Результаты и обсуждение. Проанализировано качество жизни пациентов с варикозной болезнью нижних конечностей после оперативного лечения за период с 2007 по 2016 годы. Установлено, что у подавляющего большинства пациентов (83\%) из этиологических факторов заболевания присутствовал наследственный фактор, в $57 \%$ случаев - ожирение, у 44\% - длительные статические нагрузки (44\%), у 5\% - приём гормональных контрацептивов. Дана субъективная оценка результатов хирургического лечения классическим методом (флебэктомия) и методом радиочастотной облитерации вен. Средняя продолжительность временной утраты трудоспособности железнодорожников после кроссэктомии, флебэктомии составила (18 $\pm 2,5)$ дня, в то время как после РЧА большинство пациентов (70,5\%) вернулись к привычной жизни в день операции, 23,5\% - через 2 дня после операции, 8,8\% - спустя 3 дня после операции. Показано, что своевременно проведённое оперативное лечение в плановом порядке, не дожидаясь развития осложнений варикозной болезни, значительно улучшает некоторые разделы качества жизни пациента. Заключение: процесс реабилитации больных в послеоперационном периоде в 3 раза сокращается, если оперативное вмешательство при варикозной болезни нижних конечностей проведено методом эндовенозной посегментной радиочастотной облитерации вен.
\end{abstract}

Ключевые слова: варикоз, качество жизни, радиочастотная облитерация вен

Для цитирования: Комарова Л.Н., Ряхин Р.Н., Алиев Ф.Ш., Звезда С.А. РЕЗУЛЬТАТЫ ЛЕЧЕНИЯ БОЛЬНЫХ ВАРИКОЗНОЙ БОЛЕЗНЬЮ НИЖНИХ КОНЕЧНОСТЕЙ ЗА 10 ЛЕТ. Архивъ внутренней медицины. 2018; 8(3): 215-218. DOI: 10.20514/2226-6704-2018-8-3-215-218

\begin{abstract}
The aim: to study the quality of life of patients with varicose disease of lower extremities, treated for the period from 2007 to 2016 in an operational way, including the RFA method. Materials and methods. We used the following materials in our work: medical histories of the patient of the surgical department, summary data from the department of statistics Departmental Hospital at the station Tyumen JSC «Russian Railways», data of ultrasonic angioscanization of veins of lower extremities. We used the research methods: statistical method, clinical, expert analysis and system approach. The degree of chronic venous insufficiency of lower extremities was determined using the clinical section of the international classification of CEAP (1995). Results and discussion. We analyzed the quality of life of patients with varicose disease of lower extremities after surgical treatment for the period from 2007 to 2016. It was found that the overwhelming majority of patients (83\%) had a hereditary factor from the etiologic factors of the disease, obesity in $57 \%$ of cases, long-term static loads in $44 \%$, and hormonal contraceptives in $5 \%$ of cases. We gave a subjective evaluation of the results of surgical treatment with the classical method (phlebectomy) and the method of radiofrequency obliteration of veins. The average duration of temporary disability of railwaymen after crossectomy and phlebectomy was $(18 \pm 2,5)$ days, while after the RFO the majority of patients
\end{abstract}

*Контакты/Contacts. E-mail: lnkomarova@mail.ru 
(70.5\%) returned to their usual life on the day of the operation, $23.5 \%$ after 2 days after surgery, $8.8 \%-$ after 3 days after the operation. It is shown that the promptly conducted operative treatment in a planned manner, without waiting for the development of complications of varicose veins, significantly improves some sections of the patient's quality of life. Conclusion: the process of rehabilitation of patients in the postoperative period is reduced 3 times if surgical intervention with varicose disease of lower extremities is performed by the method of endovenous segmental radiofrequency obliteration of veins.

Key words: varices, quality of life, radiofrequency obliteration of the veins

For citation: Komarova L.N., Ryakhin R.N., Aliev F.S., Zvezda S.A. TREATMENT RESULTS OF PATIENTS WITH VARICOSE VEINS DISEASE FOR TEN YEARS. The Russian Archives of Internal Medicine. 2018; 8(3): 215-218. [In Russian]. DOI: 10.20514/2226-6704-2018-8-3-215-218

DOI: 10.20514/2226-6704-2018-8-3-215-218

БПВ - большая подкожная вена, РЧО — радиочастотная облитерация, ТН — трофические нарушения, ТЯ — трофические язвы, ХВН хроническая венозная недостаточность, ЭПРЧО - эндовенозная посегментная радиочастотная облитерация

\section{Актуальность}

Хроническим заболеванием вен нижних конечностей страдает более $20 \%$ населения мира. В России (по данным М.А. Ханевич, 2003 г.) - более 35 миллионов, из них - до 35\% - трудоспособное население, более 50\% людей пожилого и старческого возраста. Распространенность трофических нарушений (ТН) и трофических язв (ТЯ) при ХВН составляет $5-8 \%$, при этом частота венозных трофических язв с возрастом увеличивается и достигает максимума после 60 лет (Кириенко А.И., 2007). Наиболее радикальным и эффективным методом лечения варикозной болезни по праву считается хирургическое вмешательство (Лосев Р.З., 2005). В настоящее время разнообразные методики хирургического лечения остаются одним из эффективных инструментов в борьбе с варикозным расширением вен. Своевременно выполненная операция позволяет не только устранить все проявления варикоза, но и предупредить развитие опасных осложнений этого заболевания. Хирургические методики лечения варикозной болезни, которые мы использовали на базе НУЗ «Отделенческой больницы на ст. Тюмень ОАО «РЖА» - это комбинированная флебэктомия, инвагинационная венэктомия, ЭХО-склерооблитерация магистральных подкожных вен, а также - эндовенозная посегментная радиочастотная облитерация (РЧО) вен. Последний метод используется наиболее активно с 2016 года, и уже зарекомендовал себя как альтернатива кроссэктомии и стриппингу. Радиочастотную облитерацию вен выполняют исключительно под ультразвуковым контролем от момента пункции вены до завершения процедуры. Под действием радиоволн происходит нагревание варикозной вены, она спадается и впоследствии замещается соединительной тканью. Аанная процедура имеет много достоинств: проводится под местной анестезией, не требует длительного нахождения в стационаре, малотравматична, оставляет косметический эффект.

Цель исследования: изучить качество жизни больных варикозной болезнью нижних конечностей, пролеченных за период с 2007 по 2016 годы оперативным путём, включая метод РЧО.

\section{Материал и методы исследования}

В работе были использованы следующие методы исследования: статистический метод, клинический, экспертный анализ и системный подход. Степень хронической венозной недостаточности нижних конечностей определяли с использованием клинического раздела международной классификации СЕАР (1995). Следует отметить, что у подавляющего большинства $(77,8 \%)$ пациентов была выявлена II сте-

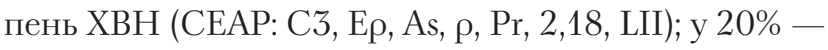
III степень.

\section{Результаты и обсуждение}

Нами проведён ретроспективный анализ историй болезни 861 больного (из них 378 железнодорожников и 483 не являются железнодорожниками). Из всех пролеченных пациентов работники железнодорожного транспорта составили 44,7\%, подавляющее большинство из которых - женщины (206 $52,7 \%)$; процент мужчин составил 47,3. По профессиональному статусу распределение варикозной болезни среди мужчин-железнодорожников было следующим: в 20\% случаях - разнорабочие, $13 \%$ (51 человек) - слесари по ремонту подвижного состава. Группа машинистов, водителей и их помощников составили 14,3\%. Среди женщин - работников железнодорожного транспорта распределение по социально-профессиональному статусу было следующим: диспетчерско-операторская группа (20\%), группа, обслуживающая поезда в пути следования (проводник пассажирского поезда, проводник по сопровождению грузов и спецвагонов и др.) - 18\%, остальную часть составили работники группы пути.

Средний возраст прооперированных больных 51,1 год, при этом минимальный возраст -22 года, максимальный - 83. Количество проведенных операций на одной нижней конечности - 605 (70,3\%), на двух - 256 (29,7\%). Из этиологических факторов у подавляющего болышинства пациентов выявлен наследственный фактор (83\%), далее по частоте 
встречаемости: ожирение (57\%), длительные статические нагрузки (44\%), приём гормональных контрацептивов (5\%). Алительность заболевания в среднем составила 11,8 лет. По данным ультразвукового сканирования вен максимальный диаметр большой подкожной вены (БПВ) был 27 мм, минимальный диаметр БПВ - 12 мм.

Основным методом инструментальной диагностики, выполненным во всех случаях для уточнения локализации, характера и протяженности патологии в венозной системе, было ультразвуковое дуплексное сканирование вен нижних конечностей. Количество сопутствующих заболеваний, а также их выраженность обуславливали тяжесть общего состояния пациента. Оценку степени риска операции и анестезии проводили с использованием классификации Американского общества анестезиологов (ASA) по тяжести соматического состояния. Оперативное лечение у 2/3 больных проводилось под спинномозговой анестезией и под внутривенным наркозом. Эндовенозная посегментная радиочастотная облитерация (ЭПРЧО) варикозно-расширенных вен нижних конечностей проведена у 68 пациентов преимущественно под местной анестезией (создавалась (паравазальная тумесцентная подушка) с использованием специальных интродьюсеров компании VNUS и под контролем УЗИ). Коагуляция вены осуществлялась с помощью радиочастотного катетера, с отступом 2 см от сафено-феморального и/или сафено-поплитеального соустья. В 36\% случаев оперативных вмешательств РЧО дополнялась диссекцией несостоятельных перфорантных вен из мини-доступа. Объем вмешательства определяли с учетом степени хронической венозной недостаточности пораженной нижней конечности и тяжести общего состояния пациента, а также учитывая выраженность воспалительного инфильтрата в зоне тромбированных варикозных вен. Противопоказанием к хирургическому вмешательству был крайне высокий операционноанестезиологический риск, обусловленный декомпенсацией сахарного диабета и выявленный у двоих пациентов старческого возраста.

Следует отметить, что превалирующими операциями до внедрения метода ЭПРЧО в клинику хирургического отделения были: кроссэктомия, инвагинационная венэктомия подкожной вены бедра с последующей окклюзией большой подкожной вены голени; перевязка перфорантных вен из минидоступа по Мюллеру, лигатурная диссекция подкожных и перфорантных вен голени и бедра. С 2016 года в протоколах операций преобладает ЭПРЧО в комбинации с минифлебэктомией и склеротерапией.

Из общего числа прооперированных больных в ближайшем послеоперационном периоде со стороны вмешательства у 29 человек была определена послеоперационная паховая гематома, в отдалённом периоде 11\% пациентов стали отмечать нарушение тактильной чувствительности. Средняя продолжительность временной утраты трудоспособности железнодорожников после оперативного лечения по поводу варикозной болезни составила $(18 \pm 2,5)$ дня, в то время как после РЧО большинство пациентов (70,5\%) вернулись к привычной жизни в день операции, 23,5\% - через 2 дня после операции, 8,8\% спустя 3 дня после операции.

Оценивая качество жизни больных после проведённой операции на венах, мы использовали в своей работе опросник CIVIQ, включающий такие критерии, как выраженность болевых ощущений, ограничение в работе или в повседневной жизни, нарушение сна, ограничение физического и социального функционирования. В результате оказалось, что 17\% больных после классической флебэктомии испытывают ограничения в физическом и социальном функционировании, 12\% - подавление психоэмоционального фона, в то время как после оперативного вмешательства методом РЧО 53\% прооперированных больных не испытывают никаких ограничений в физическом и социальном функционировании, 73\% уверяют, что (даже не поняли, что перенесли операцию), абсолютно никаких ухудшений в психоэмоциональном фоне. Одновременно всем пациентам было предложено дать собственную оценку результатов хирургического лечения, ориентируясь на успешность устранения варикозного и отёчного синдромов, а также динамику таких признаков ХВН, как боль, тяжесть, утомляемость в ногах, ночные судороги. Подавляющее большинство больных после ЭПРЧО (65,0\%) оценили эффект выполненной операции, на (отлично), отметив устранение варикозного синдрома, уменьшение отёков, ночных судорог, болевого синдрома, исчезновение (или уменьшение) трофических расстройств на голени. Ни один пациент не оценил результаты повторного хирургического лечения как неудовлетворительные.

\section{ВЫводы}

Таким образом, эндовенозная посегментная радиочастотная облитерация (ЭПРЧО) вен нижних конечностей является наиболее современным и малоинвазивным методом, значительно снижающим периоперационный стресс в предоперационном периоде и повышающим качество жизни у подавляющего числа пациентов.

Конфликт интересов и финансовое обеспечение/Conflict of interest and financial security Статья подготовлена при поддержке компании MEAТРОНИК, которая не оказывала влияние на обработку и конечные результаты работы/The article was prepared with the support of MEDTRONIK, which did not influence the processing and final results of the work 


\section{Список литературы/ References:}

1. Кириенко, А.И. Хронические заболевания вен нижних конечностей у работников промышленных предприятий Москвы (результаты 130 эпидемиологических исследований). Ангиология и сосудистая хирургия. 2004; 10(10): 77-86.

Kirienko A.I. Chronic diseases of veins of lower extremities in workers of industrial enterprises in Moscow (the results of 130 epidemiological studies). Angiology and vascular Surgery. 20042004; 10(10): 77 - 86 [In Russian].

2. Комарова Л.Н., Долгинцев В.И., Алиев Ф.Ш.. Медико-социальные аспекты долгожительства и диспансеризация больных хирургическими заболеваниями старческого возраста и долгожителей. (Монография). Тюмень. 2012; 86 с.

Komarova L.N., Dolgintsev V.I., Aliev F.Sh. Medical and social aspects of longevity and clinical examination of patients with surgical diseases of senile age and long-livers. (Monograph). Tyumen. 2012; 86 p. [In Russian].

3. Комарова Л.Н. и др. Некоторые результаты лечения гериатрических больных с хроническим заболеванием вен нижних конечностей. Академический журнал Западной Сибири. 2012; 1: 29. Komarova L.N. Some results of treatment of geriatric patients with chronic venous disease of lower extremities. Academic Journal of Western Siberia. 2012; 1: 29 [In Russian].

4. Комарова Л.Н. Варикозная болезнь вен нижних конечностей у работников железнодорожного транспорта ст. Тюмень. Материалы XI научно-практической конференции Ассоциации флебологов России. Новосибирск. Флебология. 2016; 10(2):19.
Komarova L.N. Varicose veins of lower extremities in workers of railway transport of Tyumen station. [Materials of the 11th scientific and practical conference of the Russian phlebology association]. Novosibirsk. Phlebology. 2016; 10(2):19 [In Russian].

5. Комарова Л.Н. Острый варикотромбофлебит у железнодорожников станции Тюмень. Материалы XI научно-практической конференции Ассоциации флебологов России. Новосибирск. Флебология. 2016; 10(2): 59.

Komarova L.N. Acute varicothrombophlebitis in railwaymenof Tyumen station. [Materials of the 11th scientific and practical conference of the Russian phlebology association]. Novosibirsk. Phlebology. 2016; 10(2):59 [In Russian].

6. Практикум по лечению варикозной болезни /под ред. Г.Д. Константиновой. М.: ПРОФИЛЬ. 2006; 250 с.

Practical studies of the treatment of varicose veins (Russ. ed.: Constantinova G.D.) M.: PROFILE. 2006; 250 p. [In Russian].

7. Комарова Л.Н. Современные проблемы в гериатрической хирургии и пути их решения. Тюмень. Университетская медицина Урала. 2016; 2(4): 13-15.

Komarova L.N. Modern problems in geriatric surgery and ways of their solution. Tyumen. University medicine of the Urals. 2016; 2(4): 13-15 [In Russian].

Статья получена/Article received 05.02.2018 г. Принята к публикации/ Adopted for publication 20.03.2018 г.

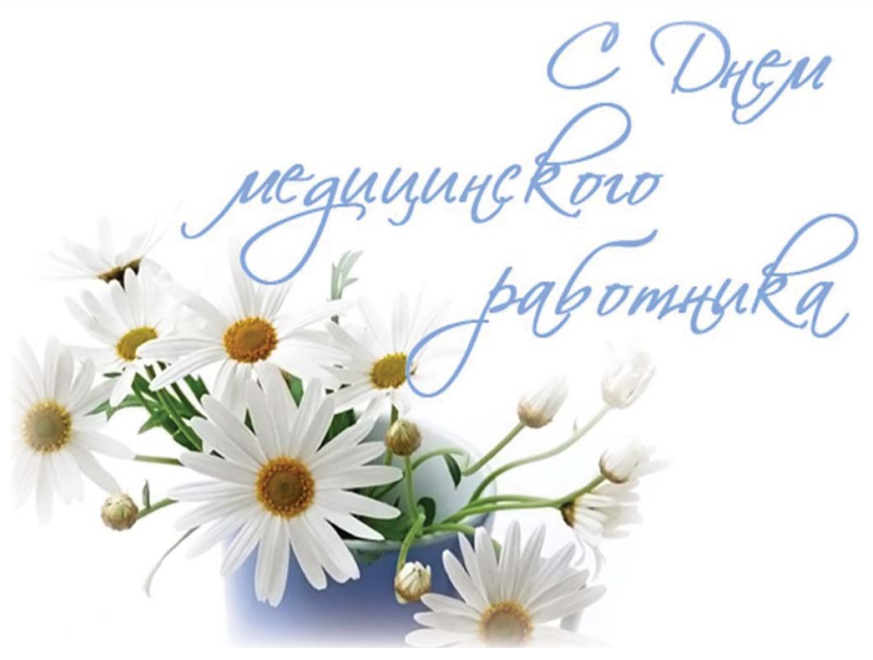

Нет на свете профессии более благородной и важной, чем профессия доктора.

Уважаемые врачи и все сотрудники больниц и поликлиник, примите искренние поздравления с профессиональным праздником с Днем медицинского работника! Пусть ваш опыт, знания и умение возвращают пациентам самую большую ценность - здоровье!

Желаем вам только позитивных эмоций и радостных событий в жизни! 